\title{
Application of ERAS Concept Combined with Psychological Stress Intervention in Laparoscopic Urological Surgery Nursing
}

\author{
Liumei Luo*, Xiangling Jiang, Xinli Kang, Fanchang Zeng, Yuzhu Lin, Dingying Wu \\ Hainan General Hospital, Haikou, China \\ Email: ^g2002m@163.com
}

How to cite this paper: Luo, L.M., Jiang, X.L., Kang, X.L., Zeng, F.C., Lin, Y.Z. and Wu, D.Y. (2021) Application of ERAS Concept Combined with Psychological Stress Intervention in Laparoscopic Urological Surgery Nursing. Health, 13, 134-143.

https://doi.org/10.4236/health.2021.132012

Received: January 17, 2021

Accepted: February 15, 2021

Published: February 18, 2021

Copyright $\odot 2021$ by author(s) and Scientific Research Publishing Inc. This work is licensed under the Creative Commons Attribution International License (CC BY 4.0).

http://creativecommons.org/licenses/by/4.0/ (c) (i) Open Access

\begin{abstract}
Enhanced recovery after surgery (ERAS), a series of evidence-based optimization measures during the perioperative period, to reduce patients' physical and psychological traumatic stress responses, to reduce complications, to shorten the length of hospital stay, to reduce the risk of readmission and mortality, and ultimately to promote rapid patient resuscitation, is adopted. The negative emotional experience of patients may aggravate the surgical stress response, interfere with the endocrine system and nervous system, cause the imbalance of internal environment, and have a negative impact on the surgical effect. This paper uses the concept of ERAS in combination with psychological stress interventions, perioperative psychological nursing instruction, reduces the laparoscopic surgery in patients with psychological stress reaction on the immune function of cells, improves the body's immune function, improves the body of bacteria such as vitamin attack resistance, reduces the risk of complications such as infection, and accelerates the process of patient rehabilitation. Here is the report.
\end{abstract}

\section{Keywords}

Accelerated Rehabilitation Surgery, Laparoscopic Urinary Surgery, Nursing, Stress Response, Psychological Intervention

\section{Introduction}

Danish surgeon Kehlet proposed the concept of enhanced recovery after surgery (ERAS) for the first time in 1997 for the comprehensive optimization of perioperative management measures [1]. In recent years, this concept has been widely promoted in European and American countries [2] and gradually adopted and ap- 
plied in China [3]. Enhanced recovery after surgery (ERAS), a series of evidence-based optimization measures during the perioperative period, to reduce patients' physical and psychological traumatic stress responses, to reduce complications, to shorten the length of hospital stay, to reduce the risk of readmission and mortality, and ultimately to promote rapid patient resuscitation, is adopted [4]. At present, ERAS has been successfully applied to many fields such as general surgery, thoracic and cardiac surgery, orthopedics, gynecology and urology, etc., attracting extensive attention and high attention from the academic community [5]. In recent years, the concept and method of accelerated rehabilitation surgery have been applied in China and achieved good results, but there are few reports on its application in urology. At the same time, the concept of rapid rehabilitation surgery is to reduce the incidence of postoperative complications and shorten the length of hospital stay of patients by nursing staff to optimize nursing measures during the perioperative period of patients, so as to promote the rapid recovery of patients [6]. With the continuous improvement of laparoscopic equipment and techniques, laparoscopic minimally invasive surgery has been widely used in the field of urology surgery. There are many types of urological diseases, most of which need surgical treatment. Surgical treatment is a certain traumatic treatment measure, and the lack of understanding of surgery and the uncertain effect of surgery will increase the adverse emotional reactions of patients. Although laparoscopic surgery has the advantages of minimally invasive, aesthetic, etc., it will still bring some damage to patients, cause anxiety, tension and other psychological discomfort symptoms, and even affect the smooth implementation of the operation and the treatment effect [7]. Chronic stress refers to the long-term Chronic stress state caused by stressors [8]. Long-term chronic stress can lead to diseases of the mental, cardiovascular and digestive systems, and also regulate the occurrence and development of cancer [9]. Diagnosis and follow-up treatment of malignant tumors can bring long-term psychological stress stimulation to patients, and most of tumor patients have negative emotions such as anxiety, fear and despair, and the long-term existence of these stressors makes patients with urinary tract tumors in a state of chronic stress [10]. Long-term chronic stress has been proved to accelerate the progression of cancer and promote the metastasis of urinary tract tumors by activating the Sympathetic nervous system (SNS) [11]. Whether long-term chronic stress in patients with urinary tract tumors affects the prognosis of patients by regulating SNS and thereby promoting tumor metastasis [12]. Therefore, it is particularly necessary to pay attention to the psychological state of patients before and after surgery, implement psychological intervention measures, and provide psychological guidance to patients during the perioperative period, so as to promote the smooth operation and achieve the best treatment effect [13].

At present, the influence of psychophysiological factors and psychosocial factors on the occurrence, development, outcome and prognosis of diseases has become a research hotspot. Moreover, the regulatory effects of psychological stress 
and neuroendocrine on urinary tract tumor metastasis and their molecular mechanisms will open a new breakthrough for the exploration of therapeutic strategies for the prevention and treatment of urinary tract tumor metastasis and recurrence. Therefore, to explore the safety of accelerated rehabilitation concept in the field of urology laparoscopic perioperative psychological stress nursing intervention, objective analysis and comprehensive evaluation of its clinical application value is a research hotspot, has a certain economic value and social value.

\section{Optimization of Preoperative Preparation}

Optimized preparation before surgery can make patients have good psychological and physiological conditions, which is the premise of rapid recovery after surgery.

\subsection{Preoperative Education and Psychological Counseling}

Most patients have different degrees of fear and anxiety before operation, fear and anxiety will cause adverse effects on patients psychologically and physically, and trigger the stress response of the body, damage the stability of the body's internal environment, increase the incidence of adverse events during the hospital. Individual preoperative education and psychological counseling are independent factors for the success of ERAS [14]. The goal of preoperative education and psychological counseling is to eliminate or reduce the tension and anxiety of patients, build up confidence, accept the operation in a healthy state of mind, and actively cooperate with the completion of perioperative treatment. The medical personnel who carry out preoperative education should have a high sense of responsibility and sympathy, and have rich knowledge of disease, operation and perioperative management, as well as certain psychological and social knowledge. To gain the trust of patients and their families is the premise of high-quality preoperative education and psychological counseling. Medical staff should introduce relevant knowledge of perioperative treatment and various measures and suggestions for promoting rapid postoperative recovery to patients and their families in various forms before operation [15], including: 1) informing patients of anesthesia and the procedure of surgery to reduce patients' fear and anxiety about anesthesia and surgery; 2) Inform patients of the purpose and main items of ERAS program, encourage patients to eat early after surgery, early after surgery activities, publicize pain control and respiratory therapy and other related knowledge, and increase the compliance of the program implementation; 3) Inform patients of the preset discharge criteria; 4) Inform patients of follow-up schedule and readmission route.

\subsection{Nutrition Screening and Nutrition Support to Shorten the Time of Water Fasting before Surgery}

Malnutrition is an independent factor affecting postoperative complications [16]. The standards of the European Association of Nutrition and Metabolism were used to determine whether patients had nutritional risks, and for patients 
with severe nutritional risks, individualized nutritional support objectives and approaches should be developed during perioperative period [15]. The purpose of fasting and abstaining from water before surgery is to ensure the full empfication of gastric contents and to avoid reflux and aspiration during anesthesia and surgery. Studies in recent years have proved that fasting and water abstaining for a long time before surgery will lead to thirst and hunger of patients, which will put patients in a state of metabolic stress, lead to insulin resistance and immune dysfunction, increase the incidence of postoperative complications, and become an important factor affecting the rapid recovery of patients after surgery [17].

\subsection{Preoperative Preparation and Risk Prevention}

Due to the complexity of retroperitoneal laparoscopic operation, sometimes different surgical instruments need to be replaced frequently during the surgical process. Therefore, the hand washing nurse on the table should be skilled in the loading and unloading and function of laparoscopic instruments to ensure that they are in a good working state. At the same time, prepare the lens to be rinsed with hot saline at $60^{\circ} \mathrm{C}-80^{\circ} \mathrm{C}$ to prevent lens atomization from making the surgical field unclear and affecting the operation. Urological organs are mostly located in the retroperitoneal area, which is concealed, and there is no natural surgical operation space, and the intraoperative anatomical marks are not clear. The hand washing nurse should be familiar with the anatomical level of the retroperitoneal approach operation, be familiar with the surgical procedures, accurately provide the instruments required during the operation, and provide conditions for doctors to shorten the operation time as far as possible. Like all laparoscopic urological surgery, retrolaparoscopic urological surgery has large blood vessel injury resulting in massive bleeding; Peripheral organ injury; Subcutaneous emphysema; Wound infection; Retroperitoneal hematoma and respiratory acidosis and other complications. Especially when intraoperative bleeding is difficult to control, the lesion site is not easily exposed and the pleura are damaged, it is highly possible to transfer to open surgery. Therefore, the relevant instruments and dressings for open surgery should be prepared in advance, and the operation should be opened in time once necessary to reduce unnecessary bleeding and waiting time in the process of transfer. Due to the application of pneumoperitoneal machine and limited surgical field in the process of retroperitoneal laparoscopic surgery, the operator is often easy to ignore some complications outside the surgical field, such as subcutaneous emphysema, accidental bleeding caused by pneumoperitoneal needle puncture and so on. Therefore, intraoperative anesthesia should be assisted to closely observe the changes in the condition, pay attention to the changes in blood pressure, heart rate and oxygen saturation and other vital signs, as well as the occurrence of subcutaneous emphysema, and timely report to the doctor to deal with the problem, so as not to delay the rescue opportunity, causing serious consequences. Surgery will bring patients a certain sense of pain, the 
appearance of pain will largely make patients have negative emotions, is not conducive to postoperative rehabilitation of patients. Therefore, nursing staff should actively communicate and communicate with patients in the process of nursing, inform patients why they feel pain and how long the pain may last, and help patients relax. In addition, caregivers can also use attention shifting therapy and psychotherapy to reduce pain, tension and anxiety.

\section{Optimization of Intraoperative Management}

Optimized management during operation can keep the function of patients' important organs and internal environment stable, which is the basis of rapid recovery after operation.

\subsection{Optimization of Anesthesia}

According to the requirements of the operation, the optimal anesthesia method can be selected, including general anesthesia, nerve block anesthesia, local anesthesia and combined application of multiple anesthesia methods. ERAS calls for minimizing the use of general anesthesia and recommends nerve block anesthesia and local anesthesia. Choose general anesthetic with quick effect and short action time, and reduce the dosage of general anesthetic as far as possible, so that patients wake up as soon as possible after general anesthesia. The use of ventilator after general anesthesia will increase the lung injury, and also increase the body's stress response, reduce the use of ventilator can reduce the lung injury and stress response.

\subsection{Warm during Operation}

After general anesthesia, the body is in a state of hypothermia, which is a normal pathophysiological reaction. However, hypothermia can cause peripheral vasoconstriction, decrease peripheral blood flow and tissue perfusion, inhibit the immune system function of the body, and increase the release of various inflammatory mediators. Multiple Meta-analyses and clinical randomized controlled studies [18] have shown that avoiding intraoperative hypothermia can reduce the incidence of incision infection, cardiac complications, bleeding and blood transfusion. In addition, intraoperative hypothermia may affect the pharmacology and pharmacokinetics, as well as anesthesia resuscitation. ERAS requires that surgical heat preservation should be strengthened to maintain room temperature $>29.5^{\circ} \mathrm{C}$ to reduce unnecessary tissue exposure during surgery. Heating device should be used for infusion, and warm saline or warm distilled water should be used to flush the wound cavity to keep the central body temperature at more than $36^{\circ} \mathrm{C}$ [15].

\subsection{Infusion Management}

Perioperative patients with reasonable transfusion can maintain blood circulation, improve tissue perfusion, stable internal environment, transfusion management a significant role in the optimization measures of ERAS, which should 
avoid tissue hypoperfusion caused by low blood volume and organ damage, also should pay attention to the capacity of the load caused by excessive tissue edema and cardiac load increase. The traditional view is that the loss of the third space is part of the fluid therapy, resulting in the "open fluid therapy" model to ensure adequate circulation volume. Studies have shown that in some patients, this "open fluid therapy" mode may lead to volume overload and increased intra-vascular hydrostatic pressure, which may promote atrial natriuretic peptide release. Natriuretic peptides can destroy the villi layer, which is formed by the polysaccharide protein complex on the surface of capillary endothelium, which plays an important role in preventing extravasation of fluid. Fluff layer destruction and intravascular hydrostatic pressure rise, can cause tissue edema, damage tissue oxygenation, affect heart, lung and gastrointestinal function, so some scholars put forward "restrictive fluid therapy" model, to physiological needs and perioperative dominant loss as the basis of fluid therapy, but restrictive fluid therapy and sometimes leads to bad circulation capacity insufficiency and tissue perfusion. Target-oriented fluid therapy is currently recognized as a scientific perioperative volume management mode, which is based on the individual volume status and fluid demand. Its core is to continuously monitor the volume status of the body with specific and sensitive monitoring indexes of circulating volume, and guide fluid therapy accordingly [19] [20].

\subsection{Reduce Surgical Trauma and Postoperative Stress Reaction}

Stress response is the response of neuroendocrine system to various adverse stimuli, which can affect the functions of multiple organs and systems. Reducing surgical trauma is the core principle of ERAS concept, and is also the basis of rapid postoperative recovery of patients. Accurate, minimally invasive and rapid surgical trauma reduction is the basic strategy. Stress can lead to the activation of pro-inflammatory factors such as interleukin 6 and induce systemic inflammatory response syndrome. Severe systemic inflammatory response syndrome is closely related to the poor prognosis of patients. Glucocorticoid, Ulinastatin and other drugs regulate inflammatory response [21]. Perioperative use of glucocorticoids is helpful to reduce surgical stress and fatigue, thus promoting recovery. However, whether perioperative use of glucocorticoids increases the risk of poor wound healing, stress gastric mucosal lesions, hyperglycemia, and infection has not been determined.

\section{Optimization of Postoperative Management}

Optimized post-operation management can make the function of the patients' important organs recover as soon as possible, and it is an important measure for the rapid recovery of patients after operation.

\subsection{Postoperative Diet Management}

Fasting and water prohibition can slow gastrointestinal peristalsis and cause ga- 
strointestinal discomfort. Early intake and water intake can promote the recovery of intestinal function. Prolonged perioperative fasting and water deprivation can also lead to dehydration, hypovolemia and internal environmental disorders. Early oral intake and water intake after surgery are conducive to the recovery of gastrointestinal function and the improvement of systemic nutritional status [22]. At present, the restoration of bowel sounds and the removal of exhaust defecation are no longer the criteria for the relief of intestinal paralysis and the resumption of oral feeding.

\subsection{Postoperative Activity Management}

Long-term bed rest will increase the incidence of pulmonary infection, embolism and other complications. Early activity can promote the recovery of multi-system functions such as musculoskeletal system and respiratory system, prevent pulmonary infection, bedsore and deep vein thrombosis, and promote the recovery of gastrointestinal function. The achievement of early activity goals depends on preoperative education, multimodal analgesia and early removal of drainage tubes. Therefore, early activities with reasonable planning are safe and beneficial. It is recommended to establish a daily activity goal and increase the activity level day by day after operation.

\subsection{Postoperative Pain Management}

Postoperative pain is an important factor affecting the process of postoperative rehabilitation. Postoperative pain can not only bring physical pain and psychological anxiety to patients, but also affect gastrointestinal function, cardiopulmonary function, neurological function, endocrine and metabolic function of patients, and directly affect the process and quality of postoperative rehabilitation of patients [23]. From the domestic clinical practice, postoperative analgesia is widespread, the main reason is that the clinicians do not pay enough attention to it. Pain treatment is an important part of ERAS, and painless surgery is regarded as one of the ultimate goals of ERAS [24]. The goals of postoperative pain treatment are: 1) good analgesic effect; 2) Minor adverse reactions and complications; 3) maintain good organ function; 4) It is conducive to postoperative rehabilitation of patients; 5) High cost performance. A post-operative acute pain management team composed of anesthesiologists, surgeons, pharmacists and nursing staff is established to optimize pain management, speed up the rehabilitation process and improve the quality of rehabilitation. Multi-mode analgesia is the combination of different drugs and different analgesic methods to reduce the use of opioids, which has the advantages of better analgesia effect and fewer side effects. The new ideas for analgesia are: 1) giving drugs on time, not on demand; 2) multimodal analgesia, rather than opioids alone; 3) Early analgesia and continuous analgesia, and single postoperative analgesia and analgesia blank period. 4) Regional analgesia is the cornerstone of multimodal analgesia. 


\section{Look}

The core of ERAS is a series of optimized perioperative management measures proven by evidence-based medicine to reduce psychological and physical traumatic stress responses, reduce complications, shorten the length of hospital stay, and reduce the risk of readmission and death. The ultimate goal is to promote rapid recovery of patients. The implementation of the ERAS concept is a systematic project, involving all aspects of diagnosis and treatment activities. It advocates the establishment of a standardized management team with the participation of surgeons, anesthesiologists, nurses, physiotherapists and even psychological experts, and sets clear and standardized goals. Professor Jiang emphasized that ERAS research should be carried out at three levels: "Tao, Dharma and Technique". "Tao" refers to ERAS's research on surgical rules, which is a theoretical innovation. "Method" refers to the research of ERAS on the process and clinical pathway, which is the innovation of management. "Technology" refers to the research on specific measures and methods by ERAS, which is the innovation of technology. It is necessary to make full use of the advantage of the large number of cases in China and launch the multi-center study of ERAS in China as soon as possible [25]. Therefore, to explore the safety of accelerated rehabilitation concept in the field of urology laparoscopic perioperative psychological stress nursing intervention, objective analysis and comprehensive evaluation of its clinical application value is a research hotspot and has a certain economic value and social value.

\section{Funding}

The work was supported by Hainan General Hospital wants the youth science and technology project (QN 202006).

\section{Conflicts of Interest}

The authors declare no conflicts of interest regarding the publication of this paper.

\section{References}

[1] Zhu, L.Y., Lu, H.J., Xu, Y.-L., et al. (2014) Stress Management Improves the Lives of Patients with Acute Myocardial Infarction. Chinese Journal of Nursing, 49, 534-539.

[2] Lirosi, M.C., Tirelli, F., Biondi, A., Mele, M.C., Larotonda, C., Lorenzon, L., et al. (2019) Enhanced Recovery Program for Colorectal Surgery: A Focus on Elderly Patients over 75 Years Old. Journal of Gastrointestinal Surgery, 23, 587-594. https://doi.org/10.1007/s11605-018-3943-2

[3] Wei, C.X., Wan, F.C., Zhao, H.W., Ma, J.J., Gao, Z.L. and Lin, C.H. et al. (2018) Application of Enhanced Recovery after Surgery in Patients Undergoing Radical Cystectomy. The Journal of International Medical Research, 46, 5011-5018. https://doi.org/10.1177\%2F0300060518789035

[4] Stoeger, S.M., Freeman, H., Bitter, B., Helmer, S.D., Reyes, J. and Vincent, K.B. (2019) Evaluation of General Surgery Residency Program Websites. American Journal of 
Surgery, 217, 794-799. https://doi.org/10.1016/j.amjsurg.2018.12.060

[5] Zhao, M.X. and Gao, L.X. (2011) Analysis of the Effect of Quality Nursing in Patients with Craniocerebral Trauma. China Journal of Practical Nursing, 27, 20-21.

[6] Lee, A.H., Young, P., Liao, R., Yi, P.H., Reh, D. and Best, S.R. (2019) I Dream of Gini: Quantifying Inequality in Otolaryngology Residency Interviews. The Laryngoscope, 129, 627-633. https://doi.org/10.1002/lary.27521

[7] Li, Q.J., Du, L., Lu, L.S., Tong, Y.F., Wu, S.B., Yang, Y.F., et al. (2019) Clinical Application of Enhanced Recovery after Surgery in Perioperative Period of Laparoscopic Colorectal Cancer Surgery. Journal of Laparoendoscopic \& Advanced Surgical Techniques, 29, 178-183. https://doi.org/10.1089/lap.2018.0708

[8] Krizanova, O., Babula, P. and Pacak, K. (2016) Stress, Catecholaminergic System and Cancer. Stress, 19, 419-428. https://doi.org/10.1080/10253890.2016.1203415

[9] Song, H., Saito, E., Sawada, N., Abe, S.K., Hidaka, A., Shimazu, T., et al. (2017) Perceived Stress Level and Risk of Cancer Incidence in a Japanese Population: The Japan Public Health Center (JPHc)-Based Prospective Study. Scientific Reports, 7, Article No. 12964. https://doi.org/10.1038/s41598-017-13362-8

[10] Surman, M. and Janik, M.E. (2017) Stress and Its Molecular Consequences in Cancer Progression. Postępy Higieny i Medycyny Doświadczalnej, 71, 485-499. https://doi.org/10.5604/01.3001.0010.3830

[11] Schmieder, A., Michel, J., Sehonhaar, K., Goerdt, S. and Schledzewski, K. (2012) Differentiation and Gene Expression Profile of Tumor-Associated Macrophages. Seminars in Cancer Biology, 22, 289-297. https://doi.org/10.1016/j.semcancer.2012.02.002

[12] Schmidt, D., Peterlik, D., Reber, S.O., Lechner, A. and Männel, D.N. (2016) Induction of Suppressor Cells and Increased Tumor Growth Following Chronic Psychosocial Stress in Male Mice. PLoS ONE, 11, e0159059. https://doi.org/10.1371/journal.pone.0159059

[13] Le, C.P. and Sloan, E.K. (2016) Stress-Driven Lymphatic Dissemination: An Unanticipated Consequence of Communication between the Sympathetic Nervous System and Lymphatic Vasculature. Molecular \& Cellular Oncology, 3, e1177674. https://doi.org/10.1080/23723556.2016.1177674

[14] Kehlet, H. (1997) Multimodal Approach to Control Postoperative Pathophysiology and Rehabilitation. British Journal of Anaesthesia, 78, 606-617. https://doi.org/10.1093/bja/78.5.606

[15] Chinese Expert Group of Accelerated Rehabilitation Surgery (2016) Chinese Expert Consensus on Perioperiative Management (2016 Edition). Chinese Journal of Surgery, 54, 413-416.

[16] Vanstijn, M.F., Korkic-Halilovic, I., Bakker, M.S., van der Ploeg, T., van Leeuwen, P.A.M. and Houdijk, A.P.J. (2013) Preoperative Nutrition Status and Postoperative Outcome in Elderly General Surgery Patients: A Systematic Review. Journal of Parenteral and Enteral Nutrition, 37, 37-43.

https://doi.org/10.1177/0148607112445900

[17] Smith, I., Kranke, P., Murat, I., Smith, A., O’Sullivan, G., Søreide, E., et al. (2011) Perioperative Fasting in Adults and Children: Guidelines from the European Society of Anaesthesiology. European Journal of Anaesthesiology, 28, 556-569. https://doi.org/10.1097/EJA.0b013e3283495ba1

[18] Kurz, A., Sessler, D.I. and Lenhardt, R. (1996) Perioperative Normothermia to Reduce the Incidence of Surgical-Wound Infection and Shorten Hospitalization. New 
England Journal of Medicine, 334, 1209-1216. https://doi.org/10.1056/NEJM199605093341901

[19] Li, J.S. and Jiang, Z.W. (2015) Clinical Significance of Enhanced Recovery after Surgery Is Not Only to Shorten the Duration of Hospital Stay. Chinese Journal of Digestive Surgery, 14, 22-24.

[20] Legrand, G., Ruscio, L., Benhamou, D. and Pelletier-Fleury, N. (2015) Goal Directed Fluid Therapy Guided by Cardiac Monitoring during High-Risk Abdominal Surgery in Adult Patients: Cost-Effectiveness Analysis of Esophageal Doppler and Arterial Pulse Pressure Waveform Analysis. Value Health, 18, 605-613.

https://doi.org/10.1016/j.jval.2015.04.005

[21] Committee of Liver Cancer of Chinese Anti-Cancer Association (2014) Multiple Disciplinary Consensus on the Management of Excessive Inflammatory Response in the Perioperative Period of Hepatectomy (2014 Edition). Chinese Journal of Digestive Surgery, 13, 51-755.

[22] Bozzetti, F. and Mariani, L. (2014) Perioperative Nutritional Support of Patients Undergoing Pancreatic Surgery in the Age of ERAS. Nutrition, 30, 1267-1271.

https://doi.org/10.1016/j.nut.2014.03.002

[23] Varadhan, K.K., Neal, K.R., Dejong, C.H., Fearon, K.C.H., Ljungqvist, O. and Lobo, D.N. (2010) The Enhanced Recovery after Surgery (ERAS) Pathway for Patients Undergoing Major Elective Open Colorectal Surgery: A Meta-Analysis of Randomized Controlled Trials. Clinical Nutrition, 29, 434-440. https://doi.org/10.1016/j.clnu.2010.01.004

[24] Oderda, G. (2012) Challenges in the Management of Acute Postsurgical Pain. Pharmacotherapy, 32, 6s-11s. https://doi.org/10.1002/j.1875-9114.2012.01177.x

[25] Jiang Z.W. and Li, J.S. (2016) Research Status of Accelerated Rehabilitation Surgery in China. Chinese Journal of Gastrointestinal Surgery, 19, 246-249. 